\title{
Effect of phosphorous on the growth, optical, mechanical and thermal properties of L-alanine crystals
}

\author{
M. Anbuchezhiyan, ${ }^{* 1}$ A. Arputhalatha, ${ }^{1,2}$ S. Ponnusamy, ${ }^{3}$ K. Syed Suresh Babu ${ }^{4}$ \\ ${ }^{I}$ Department of Physics, Valliammai Engineering College, SRM Nagar, Kattankulathur - 603 203, India, \\ ${ }^{2}$ Dep. of Physics, Prince Shri Venkaterswara Padmavathy Engineering College, Kanchipuram Dt-600127, India, \\ ${ }^{3}$ Centre for Material Science and Nano Devices, Dep. of Physics, SRM University, Kattankulathur - 603 203, India, \\ ${ }^{4}$ Department of Physics, Presidency College (Autonomous), Chennai-600 005, India.
}

Received March 27, 2015; accepted June 23, 2015; published June 30, 2015

\begin{abstract}
Single crystals of L-alanine were grown from aqueous solution with a small amount of orthophosphoric acid $\left(\mathrm{H}_{3} \mathrm{PO}_{4}\right)$. The single crystal X-ray diffraction (XRD) analysis was employed to identify the cell parameters. The grown crystal was characterized by Energy Dispersive X-ray (EDX) spectrum. The UV-visible transmittance was recorded. The presence of a functional group was confirmed by FTIR analysis. The TGA/DTA studies showed the thermal properties of the crystals. The mechanical strength was analyzed by measuring microhardness of the grown compounds. The second harmonic generation of grown crystals was confirmed by the KurtzPerry method using an Nd:YAG laser.
\end{abstract}

Nonlinear optical (NLO) materials capable of generating the second harmonic frequency play a major role in the domain of optoelectronics and photonics [1]. Due to their large nonlinearity, low cut-off wavelengths, high thresholds and short response time to laser power, NLO materials are attracting much attention for possible use in optical devices. In particular, efforts have been made on pure amino acid and their complexes like Lalanine [2], L-valine [3], $\gamma$-glycine [4], L-serine sodium nitrate [5], L-histidine tetrafluoroborate [6], L-histidine bromide [7], etc. Among the amino acids, L-alanine is the simplest molecule having second harmonic generation (SHG) efficiency, of which one third if that of well known potassium dihydrogen phosphate (KDP) [8].

Phosphoric acid as a dopant has been found to yield crystals with a large plane area useful for IR detector applications [9, 10]. Even at a very low concentration level of orthophosphoric acid, there has been reported [11] a significant improvement in the pyroelectric coefficient, lowering of dielectric permittivity at a transition temperature $\left(T_{c}\right)$ and an increase in the coercive field of the triglycine sulphate (TGS) crystal. The influence of $\mathrm{H}_{3} \mathrm{PO}_{4}$ dopant concentration on the TGS crystal growth and properties has been studied and the main objective has been to optimize the dopant concentration effective for an IR applications [12]. The presence of orthophosphoric acid in L-valine crystal exhibits improved mechanical strength and SHG [13].

\footnotetext{
*E-mail: chezhiyan70@gmail.com
}

An attempt has been made to study the effect of acids on the optical, mechanical and thermal properties of Lalanine. Current investigation has been focused the effect of $\mathrm{H}_{3} \mathrm{PO}_{4}$ on L-alanine growing from aqueous solution. The starting material was synthesized by adding orthophosphoric acid $(1 \mathrm{ml})$ to the high purity L-alanine $(2.23 \mathrm{~g})$ salt, taken and dissolved in $25 \mathrm{ml}$ of deionized water in a beaker. The mixed solution was slightly heated and stirred continuously for 8 hours. After that the saturated solution was filtered and then prepared in a crystallizing vessel with perforated covers. It was kept in a constant temperature bath in undisturbed conditions to maintain a temperature of $35^{\circ} \mathrm{C}$.

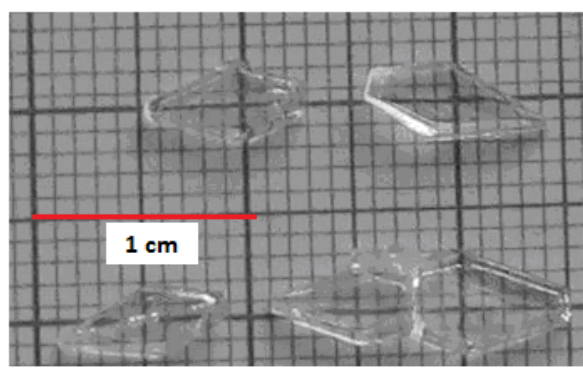

Fig. 1. Photograph of grown crystal of L-alanine in the presence of a small amount of orthophosphoric acid.

After the growth period of one week, good quality transparent crystals were obtained from the saturated aqueous solution of L-alanine in the presence of $\mathrm{H}_{3} \mathrm{PO}_{4}$.

Figure 1 shows the photograph of the grown crystal. Single crystal X-ray diffraction studies were done using a BRUKER AXS KAPPA APEX (II) CCD diffractometer with $\mathrm{Mo} \mathrm{K}_{\alpha}(\lambda=0.710693 \AA)$ radiation. It was found that the crystal of L-alanine in the presence of $\mathrm{H}_{3} \mathrm{PO}_{4}$ had the orthorhombic structure with the following lattice parameter values: $a=5.81 \AA, b=6.06 \AA, c=12.40 \AA$, and the cell volume $V=436.59 \AA^{3}$. For pure L-alanine the grown compounds cell parameters were: $a=5.779 \AA, b=6.022 \AA$, $c=12.323 \AA$ and the volume $V=428.9 \AA^{3}$ [8]. There was a very small change in lattice parameters of orthophosphoric acid doped in L-alanine crystals. The grown L-alanine crystal has an orthorhombic symmetry 
with four molecules per unit cell with the space group $\mathrm{P} 2{ }_{1} 2_{1} 2_{1}$. The molecules are found to be of the zwitter ionic nature, i.e., $\mathrm{CH}_{3} \mathrm{CH}\left(\mathrm{NH}_{3}^{+}\right) \mathrm{COO}^{-}$form and all the three hydrogen atoms of ammonium group form a hydrogen bond with three different neighbouring molecules. There is a small increase in the volume of the unit cell of the grown compound, which may be due to the incorporation of phosphoric acid molecules. Energy dispersive X-ray spectroscopy (EDX) allows one to identify what those particular elements are and their relative proportions (Weight\%). In the incorporation phosphorous into the L-alanine crystalline matrix was confirmed by EDX. The EDX spectrum of the grown crystal is shown in Fig. 2. The weight percentage (wt\%) of $\mathrm{C}, \mathrm{O}, \mathrm{P}$, and $\mathrm{Au}$ as obtained from EDX analysis for the crystal are presented in Table 1 . It is seen that a small amount of phosphorous incorporated into the host crystal.

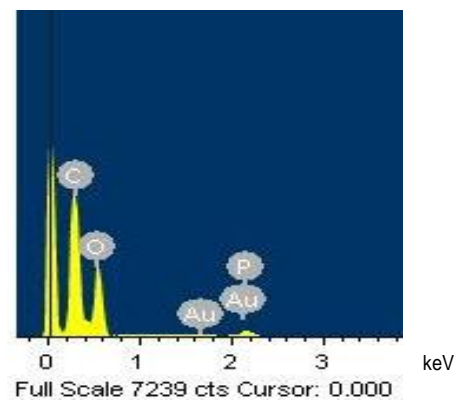

Fig. 2. Energy dispersive spectrum of L-alanine with small amount of orthophosphoric acid crystal.

The optical transmittance spectrum has been recorded using a Perkin Elmer Lambda 35 spectrophotometer in the range $190 \div 1100 \mathrm{~nm}$ covering the entire UV and Visible region. The obtained transmittance spectrum of grown compound L-alanine with a small amount of orthophosphoric acid is shown in Fig. 3. The grown compound is transparent in the visible region and there is no absorption in the entire UV-Visible region. This is a constructive nature of nonlinear optical crystals. The cutoff wavelength was observed around $238 \mathrm{~nm}$ and no absorption from $238 \mathrm{~nm}$ to $1100 \mathrm{~nm}$ and it can be used as a material for SHG and related optoelectronic applications. The cut-off wavelength is slightly lower than that of pure L-alanine reported $[8,14,15]$.

Table 1. Composition of the experimental materials wt\% (EDX elemental analysis)

\begin{tabular}{|c|c|c|}
\hline Element & Weight $\%$ & Atomic \% \\
\hline $\mathrm{C}$ & 52.44 & 62.22 \\
\hline $\mathrm{O}$ & 41.84 & 37.27 \\
\hline $\mathrm{P}$ & 0.24 & 0.11 \\
\hline $\mathrm{Au}$ & 5.49 & 0.40 \\
\hline Total & 100.00 & 100.00 \\
\hline
\end{tabular}

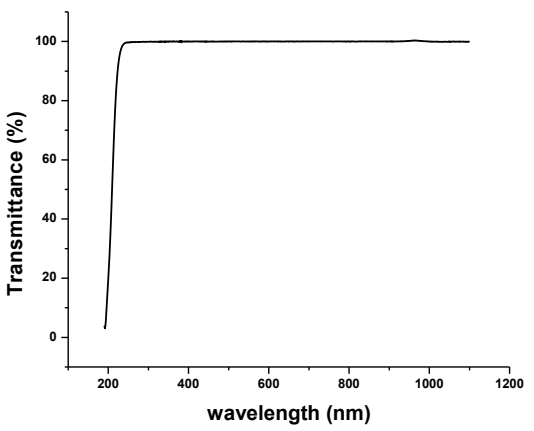

Fig. 3. UV-Visible spectrum of grown compound.

The FTIR spectrum was recorded in a range of $4000 \div 450 \mathrm{~cm}^{-1}$, using a Perkin Elmer Spectrum RX1 spectrophotometer. The FTIR spectrum of the grown Lalanine with a small amount of orthophosphoric acid crystal is shown in Fig. 4. The substitution of phosphoric acid causes the broadening of bands in the higher energy region. This is a clear indication of the presence of a small amount of phosphoric acid in the grown compound, as evident from the compositional analysis. The appearance of a broad band at $3256 \mathrm{~cm}^{-1}$ indicates the presence of protonated amino group $\mathrm{NH}_{3}{ }^{+}$stretching vibration. The symmetric bending vibrations of the ammonium group appeared at $1518 \mathrm{~cm}^{-1}$. The deprotonated carboxylate group shows a strong band near $1412 \mathrm{~cm}^{-1}$ for assigned as symmetric stretching vibration. The presence of $\mathrm{C}-\mathrm{O}$ vibration is assigned at $1113 \mathrm{~cm}^{-1}$. The peak at $1361 \mathrm{~cm}^{-1}$ and $1455 \mathrm{~cm}^{-1}$ is assigned as $\mathrm{CH}_{3}$ bending vibration. In the FTIR spectrum bands frequencies at $538 \mathrm{~cm}^{-1}\left(\mathrm{NH}_{3}{ }^{+}\right.$ torsion), and $649 \mathrm{~cm}^{-1}\left(\mathrm{CO}_{2}^{-}\right.$deformation) are observed.

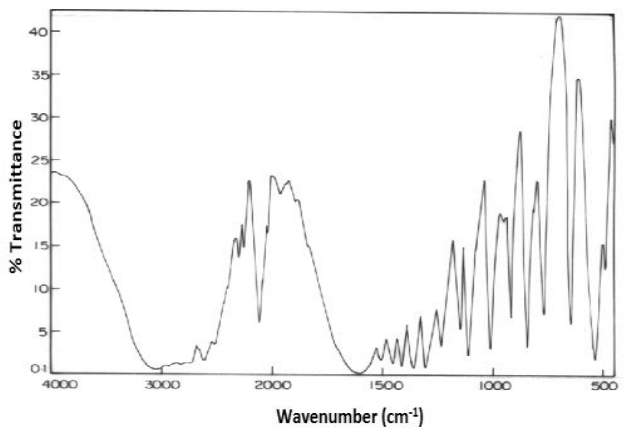

Fig. 4. FTIR spectrum of the grown compound.

The thermal stability of single crystal was estimated by Thermo Gravimetric Analysis (TGA) and Differential Thermal Analysis (DTA) techniques. Simultaneously thermo gravimetric and differential thermal analyses were carried out with the help of an instrument TG/DTA 6200 SII EXSTAR 6000 thermal analyzer. The experiment was carried out in a nitrogen atmosphere at a heating rate of $10^{\circ} \mathrm{C} / \mathrm{min}$ in the temperature range $30 \div 600^{\circ} \mathrm{C}$. A sample of $6.417 \mathrm{mg}$ was taken into a crucible. Simultaneously 
recorded TGA and DTA curves are shown in Fig. 5. From the TGA curve one can see that the material starts decomposing above $210^{\circ} \mathrm{C}$, leaving a residue. There is no water molecule in the crystal and the crystal lattice, therefore no mass loss is observed before $210^{\circ} \mathrm{C}$. The decomposition takes place within a temperature range of $210.42^{\circ} \mathrm{C}$ to $329.12^{\circ} \mathrm{C}$. The small percentage remaining corresponds to the residual carbon. The probable decomposition products are $\mathrm{NH}_{3}, \mathrm{CO}_{2}, \mathrm{CO}$ and $\mathrm{CH}_{2}=\mathrm{CH}_{2}$ (ethylene). However, the DTA thermogram clearly indicates the endothermic peak appearing at $289.3{ }^{\circ} \mathrm{C}$, which corresponds to the melting point of the sample. The melting point of a grown crystal determined with a melting point apparatus VEEGO was found to be $289^{\circ} \mathrm{C}$, which is almost equal to TGA-DTA analysis.

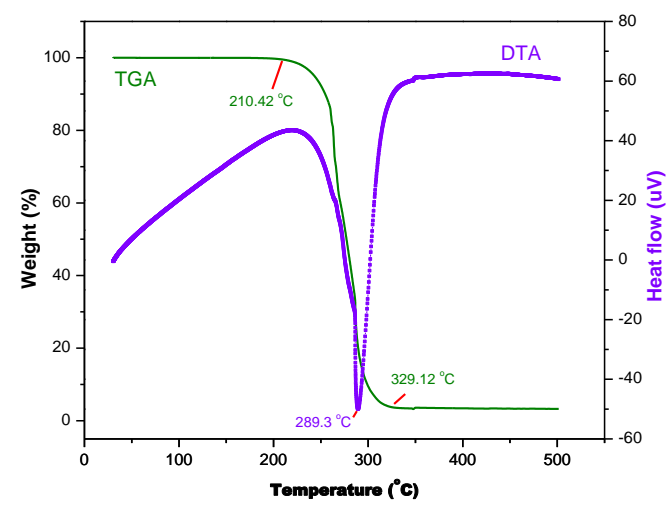

Fig. 5. TGA - DTA curve for grown crystal.

To evaluate the Vickers hardness, several indentations were made on the face of the crystal [16]. The diagonal length of the indentations was measured using a micrometer eyepiece. Dependence of the micro hardness on the load for grown crystals was evaluated. Loads of different magnitude $(25,50,100$ and 200g) were applied on L-alanine crystal for a fixed interval of time. Hardness was found to increase as the load increased. The hardness number $H_{\mathrm{v}}$ is found to be $80.9 \mathrm{~kg} / \mathrm{mm}^{2}$ for $200 \mathrm{~g}$. Beyond the load of $200 \mathrm{~g}$, a significant cracking occurs, which may be due to the release of internal stress generated by indentation. In order to find out the nonlinear optical property of the grown compound, an SHG efficiency test was performed by the Kurtz and Perry powder technique [17]. The SHG efficiency of the grown crystal was studied using a pulsed Nd:YAG laser beam of wavelength $1064 \mathrm{~nm}$, with an input power of $2.12 \mathrm{~mJ}$ and pulse width of $10 \mathrm{~ns}$, the repetition rate being $10 \mathrm{~Hz}$. The crystals were ground to a uniform particle size of about $125 \div 145 \mu \mathrm{m}$ and then packed in capillaries of uniform bore and exposed to laser radiation. The same particle size of the KDP sample was used as a reference material. The second harmonic signal generated was confirmed from the emission of green radiation (i.e. the wavelength $\lambda=532 \mathrm{~nm}$ ) from the sample. The emission of green radiation confirms the SHG in the crystal, the grown crystal was found to have the SHG as 0.14 times than that of KDP.

In conclusion, single crystals of L-alanine with a small amount of phosphoric acid were grown from an aqueous solution by the slow evaporation method. Single crystal $\mathrm{X}$-ray diffraction has been performed to determine the lattice parameters of the grown crystal. The presence of phosphorus in L-alanine is confirmed by EDX analysis. The UV-visible transmittance spectra reveal that the crystal has a cut-off wavelength at $238 \mathrm{~nm}$ and it is observed that crystal has good transparency in the visible region. The FTIR spectrum confirms the presence of functional groups. Thermal studies confirm that the crystal structure is stable up to $289.3^{\circ} \mathrm{C}$ and indicate its suitability for application in the lasers' field. A micro hardness test reveals that the grown crystal has an improved mechanical strength. The NLO property of the crystal was examined by performing the Kurtz powder test using an Nd:YAG laser. It is concluded that optical, mechanical and thermal properties of phosphoric acid doped crystals can be a material for SHG device applications.

\section{References}

[1] D.S. Chemla, J. Zyss, (Eds.), Nonlinear Optical Properties of Organic Molecules and Crystals (Academic Press, Orlando, New York, 1987).

[2] C. Razzetti, M. Ardoino, L. Zanotti, M. Zha, C. Paorici, Cryst. Res. Technol. 37, 456 (2002).

[3] E. Ramachandran, S. Natarajan, Cryst. Res. Technol. 44, 641 (2009).

[4] K. Srinivasan, J. Arumugam, Optic. Mater. 30, 40 (2007).

[5] Sd. Z. Ali Ahamed, G.R. Dillip, L. Manoj, P. Raghavaiah, B. Deva Prasad Raju , Phot. Letters Poland 2(4), 183 (2010).

[6] S. Dhanuskodi, J. Ramajothi, Cryst. Res. Tech. 39, 592 (2004).

[7] R. Ittyachan, P. Sagayaraj, J. Cryst. Growth 249, 557 (2003).

[8] M. Lydia Caroline, R. Sankar, R.M. Indirani, S. Vasudevan, Mater. Chem. Phys. 114, 490 (2009).

[9] G. Ravi, S. Anbukumar, P. Ramasamy, J. Cryst. Growth 133, 212 (1993).

[10] N. Nakatani, M. Yoshio, Jpn. J. Appl. Phys. 35, L 508 (1996)

[11] N. Nakatani, M. Yoshio, Jpn. J. Appl. Phys. 35, 5752 (1996).

[12] A. Saxena, V. Gupta, K. Sreenivas, J. Cryst. Growth 263, 192 (2004).

[13] M. Anbuchezhiyan, S. Ponnusamy, C. Muthamizhchelvan, C.C. Kanakam, S.P. Singh, P.K. Pal, P.K. Datta, Eur. Phys. J. Appl Phys. 58, 10201 (2012).

[14] K. Seethalakshmi, S. Perumal, Recent Research Sc. Techn. 4(6), 13 (2012).

[15] Ferdousi Akhtar, Jiban Podder, Research J. Physics 6(2), 31 (2012).

[16] K. Sangwal, B. Surowska, Mater. Res. Innovat. 7, 91 (2003).

[17] S.K. Kurtz, T.T. Perry, J. Appl. Phys. 39, 3798 (1968) 